\title{
Development of polyaniline-modified polysulfone nanocomposite membrane
}

\author{
Javed Alam • Lawrence Arockiasamy Dass • \\ Mansour Saleh Alhoshan - Mostafa Ghasemi • \\ Abdul Wahab Mohammad
}

Received: 18 July 2011/Accepted: 10 November 2011/Published online: 29 November 2011

(C) The Author(s) 2011. This article is published with open access at Springerlink.com

\begin{abstract}
In the present investigation, polyaniline (PANI) nanoparticles were used to improve the separation figures of merit of polysulfone (PSu) membrane. Polyaniline nanoparticles were dispersed into polysulfone matrix for the development of PSu/PANI nanocomposites through solution blending. A wet phase inversion method was used to fabricate a flat sheet polysulfone (PSu) and PSu/PANI nanocomposite membranes. The structure and characteristic properties of the membranes were investigated in terms of the surface and cross-section morphologies, roughness, and hydrophilicity, which were interpreted by scanning electron microscope, atomic force microscope, and water contact angle meter, respectively. Apart from these, the uniform dispersion of polyaniline nanoparticles $(<20 \mathrm{~nm})$ into polysulfone matrix was ascertained by transmission electron microscope. Compared with polysulfone membrane, PSu/PANI nanocomposite membranes had more hydrophilicity and smooth surface, and honeycomb crosssection structure. Therefore, the nanoparticles inclusion in the polysulfone membrane showed a significant effect on hydrophilic property as well as membrane morphology, which resulted in improvement of the permeability characteristics of polysulfone membrane.
\end{abstract}

J. Alam $(\bowtie) \cdot$ L. A. Dass · M. S. Alhoshan

King Abdullah Institute for Nanotechnology,

King Saud University, P.O. Box 2455, Riyadh 11451,

Kingdom of Saudi Arabia

e-mail: javaalam@ksu.edu.sa

M. Ghasemi - A. W. Mohammad

Department of Chemical and Process Engineering,

Faculty of Engineering and Built Environment,

University Kebangsaan Malaysia,

43600 UKM-Bangi, Selangor, Malaysia
Keywords Separation figures of merit - Hydrophilic surface $\cdot$ Phase inversion method P Permeability characteristics

\section{Introduction}

Today, membrane technology is the leading technology for all kinds of separation techniques due to its intrinsic characteristics such as operational simplicity, environmental impact, high selectivity and permeability for the transport of specific components (Mulder and Mulder 1996). It holds a significant commercial impact in several areas including water and wastewater treatment (Ashraf 2009), chemical, metallurgical and petrochemical-related industries, food industries and bioseparation areas (Baker 2004). A recent advance in membrane technology is the focus on the development of membrane materials which are the key determinants of separation performance and water productivity (Lee et al. 2011; Ulbricht 2006; Nunes and Peinemann 2010). The materials used in membrane production are generally constructed from durable polysulfone and its derivatives, the majority of which are hydrophobic. Due to hydrophobic nature of PSu material, conventional PSu membranes easily suffer serious membrane fouling and have low permeation flux, making it undesirable in the long-lasting filtration process (Higuchi et al. 1991; Ghosh and Hoek 2009). As a consequence, the surface of these membranes must be rendered hydrophilic through the addition of wetting agent, e.g. hydrophilic polymers and/or by chemical modification of the membrane structure prior to use in aqueous separations. The approaches currently used for membrane surface modification include coating, surface graft polymerization, exposure to irradiation, interfacial polymerization, and etc 
(Deng et al. 2011; Dong et al. 2009; Patel et al. 2009; Hegde et al. 2011; He et al. 2009). However, all approaches have extra processing steps to meet the goal for membrane surface modification. Breakthroughs effects of nanocomposites on the membrane performance that have been reported in the field of water and wastewater treatment include fouling mitigation, improvement of permeate quality and flux enhancement (Anadão et al. 2010; Jeong et al. 2007; Jadav and Singh 2009; Diallo et al. 2009; Hoek and Ghosh 2009).

Interest has recently developed in conducting polymers as materials for the nanocomposite membranes. Conducting polymers are a new class of polymers with unique chemical and electrochemical properties. Thermally and chemically stable conducting polymers have attracted much interest to membrane materials since they elicit the possibility of both exploiting the chemical and physical attributes of the polymer and incorporating their electronic and electrochemical properties to enhance the separation features. Also they can be used either as deposited films or nanoparticles that can be easily bound with polymers or be firmly fixed on substrate membranes through different chemical reactions. Among the family of conducting polymers, polyaniline is one of the most useful since it is environmentally stable in both its doped, conducting form and in its de-doped, insulating form. Polyaniline is also unique among conducting polymers in that it has a very simple acid/base doping/dedoping chemistry (facile and reversible electrochemistry, where the polymer can be oxidized and reduced with simultaneous change in properties). For example, undoped form of polyaniline is hydrophobic, while in the doped form polyaniline has been shown to be hydrophilic. Together, these properties make polyaniline a promising candidate for liquid separations. Several membrane systems based on polyaniline have been significantly investigated for last few decades (Liu et al. 1994; Rebattet et al. 1995; Wen and Kocherginsky 1999; Bhadra et al. 2009; Mansouri and Burford 1994; Gupta et al. 2006; Feldheim and Elliott 1992; Fan et al. 2008a, b; Deligöz 2007; Sivakumar et al. 2006). However, polyaniline has been generally categorized as an intractable polymer because of the difficulty in solution processing. The subject of many publications focused on the improvement of its processability in the past several years, such as substitution of aromatic ring of polyaniline with $-\mathrm{CH}$, - $\mathrm{OCH}$, -SO, or long alkyl chain that leads to higher solubility in organic solvents and even in water. Simultaneously, undoped polyaniline (emeraldine salt form) shows better solubility in common nonpolar or weakly polar organic solvents and surprisingly desired bulk polymers (such as polysulfone) are also dissolved in these solvents. Therefore, the development of polyaniline nanocomposites and blends via solution blending is expected to make a critical impact in fabricating higher performance membranes with increased permeability, selectivity. In the present study, a highly processible form of polyaniline (undoped polyaniline) was used as nanoparticles to develop polysulfone nanocomposites. The dispersion of polyaniline to polysulfone casting solutions was accomplished by blending process which has advantages over other membrane developing methods as it can produce surface-modified membranes via a single-step casting procedure. The novelty of this study is that all improvements (such as hydrophilicity, membrane roughness, mechanical stability and selectivity) in modified membrane are obtained at low loading of polyaniline $(\leq 0.5$ mass $\%)$. In addition, the synthesis route of a doped PSu/PANI nanocomposite membrane is different from others already mentioned in literature.

\section{Experimental}

Materials and reagents

Double distilled aniline (Aldrich), hydrochloric acid 37\% (HCl) (R\&M Chemicals), 1-methyl-2-pyrrolidone (NMP) (Aldrich), ammonium hydroxide 30\% (R\&M Chemicals) and ammonium peroxydisulphate $\left(\mathrm{NH}_{4}\right)_{2} \mathrm{~S}_{2} \mathrm{O}_{8}$ (APS, $\mathrm{R} \& \mathrm{M}$ Chemicals) were used as received. Polysulfone (Aldrich, Typical $M_{w}=35,000$ ), acetone (Bendosen laboratory chemicals), methanol (R\&M chemicals) distilled water. Polyethylene glycol (PEG, molecular weight 600-10,000) was of chemical grade and obtained from Merck. Sodium hydroxide (R\&M chemicals) and sodium chloride (SigmaAldrich) were of chemical grade and used without any further purification.

\section{Synthesis of polyaniline}

Polyaniline was synthesized (conventional route in aqueous medium using $\mathrm{HCl}$ as dopant ion) by chemical polymerization of aniline using ammonium persulfate as an oxidant (Huang and Kaner 2004). According to this route, aniline monomer, acid dopant and oxidizing agents were taken in the molecular ratio of 1:1:1.1 aniline monomer was added into the $1 \mathrm{~N}$ aqueous solution of hydrochloric acid and stirred to get aniline-acid complex and kept in the freeze to attain the reaction temperature of $0-5^{\circ} \mathrm{C}$. In another beaker $\left(\mathrm{NH}_{4}\right)_{2} \mathrm{~S}_{2} \mathrm{O}_{8}$ was dissolved in distilled water for an initiator of aniline monomer. A fixed volume of initiator was added drop wise to a $250-\mathrm{ml}$ round bottom flask containing $1 \mathrm{~N}$ $\mathrm{HCl}$ and double distilled aniline $(5 \mathrm{ml})$ with slow and continuous stirring maintained at a constant temperature. After an induction period of $30 \mathrm{~min}$, the color of the solution changed from brown to green, which confirmed 
the polymerization of aniline in conducting form. Polymerization was further continued for $12 \mathrm{~h}$ at room temperature. The green precipitate of polyaniline was filtered, and washed several times with distilled water, methanol, and then dried in vacuum for $72 \mathrm{~h}$ at $60^{\circ} \mathrm{C}$. Hereafter the green PANI powder was converted to an insulating dark blue PANI powder by deprotonation with $\mathrm{NH}_{4} \mathrm{OH}$ and then dried.

Fabrication of doped/undoped PSu/PANI nanocomposite membranes

Synthesis of PSu/PANI nanocomposites has been synthesized by solution blending with NMP. A solution of undoped polyaniline was slowly added to $20 \mathrm{wt} \%$ polysulfone solution with stirring at $55^{\circ} \mathrm{C}$ for uniform dispersion. PSu/PANI nanocomposite solution was then uniformly spread on a flat glass surface using a casting Gardner knife (Filmographe: Doctor Blade 360099003) of $0.25 \pm 0.02$ $\mathrm{mm}$ thickness. Immediately after casting, the membrane was fully immersed into a deionized water bath at room temperature for phase inversion. The membrane was then peeled off from the glass. After that, undoped PSu/PANI nanocomposite blue color membrane was transferred to water bath. The membrane was then left for $4 \mathrm{~h}$ in $0.1 \mathrm{~N}$ $\mathrm{HCl}$ solution. Once the membrane turned from dark blue to dark green, it indicated that acid doping had taken place. Then the membrane was dried in vacuum oven at $80^{\circ} \mathrm{C}$ for $6 \mathrm{~h}$ before the permeations measurement. The undoped and doped PSu/PANI nanocomposite membranes are shown in Fig. 1a, b.

\section{Membrane characterizations}

Scanning electron microscopy (SEM Gamm: modol supra 55vp-Zeiss) was used to observe the surface and crosssection morphologies of membranes. The membranes were frozen before prior to SEM observation, followed by gold sputtering (Biorad Polaron Division) (Philips SEMEDAX; XL40; PW6822/10) with the potentials of $5 \mathrm{kV}$ at different magnifications.

The uniform distribution of polyaniline nanoparticles into polysulfone host were ascertained by transmission electron micrographs (TEM Philips: $\mathrm{cm} 12$ Transmission Electron Microscope). In addition, atomic force microscopy (AFM) (using a Veeco MultiMode SPM with a Nanoscope V controller) was used to characterize the topography of membrane surfaces. All measurements were performed on dry membrane samples under ambient atmospheric conditions and the membrane surfaces were imaged in tapping mode. In addition, ATR-FTIR spectra for the composite membranes were taken on BRUBERVERTEX 80.
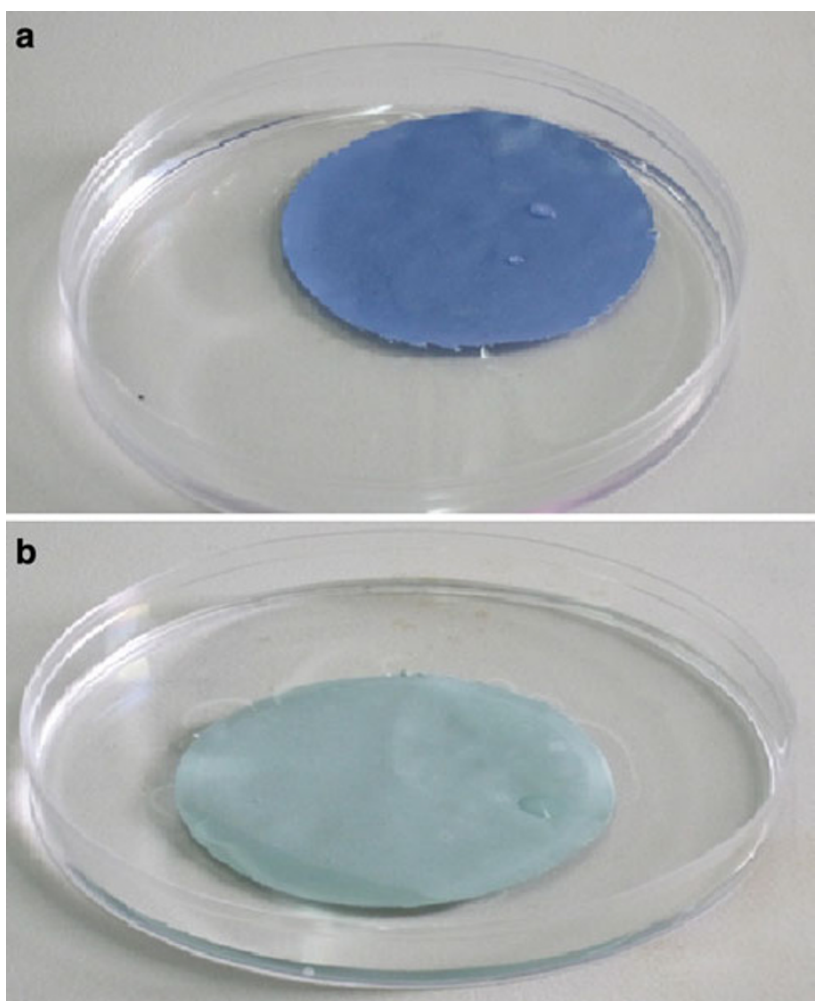

Fig. 1 Photographs of a PSu/PANI undoped, $\mathbf{b}$ doped nanocomposite membrane

Contact angles were measured with a contact angle meter (contact angle meter $110 \mathrm{VAC}, 50 / 60 \mathrm{~Hz}$ ) by sessile drop method that is where a water droplet was placed onto the membrane surface, using a syringe, and the values reported were averages of five separate measurements on the same membrane.

\section{Permeability experimental setup}

All the water permeability experiments were carried out using SEPA ST Stirred Cell (Osmonics USA). A circular membrane disk with a diameter of $0.049 \mathrm{~m}(49 \mathrm{~mm})$ was placed at the bottom of the filtration module, the active top layer towards the feed solution. The membrane was sealed between a Teflon o-ring and a stainless steel porous support. In this way, the membrane surface had an active area of $14.2 \mathrm{~cm}^{2}$. The feed solution was magnetically stirred with a Teflon-coated stirrer bar. The maximum volume capacity of the cell was $0.3 \mathrm{~L}$ and the maximum operating pressure was 10 bars. The stirring speed was fixed at $400 \mathrm{rpm}$. Pressure at the feed side was supplied by an inert gas $\mathrm{N}_{2}$. Preliminary permeation experiment was conducted for ultrapure at various ranges of separation pressure (1-6 bars) and temperature at $25 \pm 2{ }^{\circ} \mathrm{C}$.

The performances of the prepared membranes were evaluated in terms of pure water permeation flux (PWP) as 
described elsewhere (Mulder and Mulder 1996; Sabde et al. 1997). PWP and solute water permeation flux (SWP) of membrane is obtained as follows:

$J=\frac{Q}{\Delta t \times A}$

where, $J\left[\mathrm{~L} /\left(\mathrm{m}^{2} \mathrm{~h}\right)\right]$ is the permeation flux of membrane for pure water, $Q$ is the volume of permeate solution $(\mathrm{L}), \Delta t$ is permeation time (h) and $A$ is membrane area in $\mathrm{cm}^{2}$. All the flux results were triplicate.

Solute rejection of the prepared membranes were evaluated with various molecular weight PEG solutions ranging from $600-10,000 \mathrm{Da}$ at 5 bar. The concentration of PEG solution used is $500 \mathrm{ppm}$. The concentration of the feed and permeate solution were determined by the method described as follows:

Reagent A: $5 \%(\mathrm{w} / \mathrm{v}) \mathrm{BaCl}_{2}$ in $1 \mathrm{~N} \mathrm{HCl}(100 \mathrm{ml})$

Reagent B: 2\% (w/v) KI diluted 10 times $+1.27 \mathrm{~g} \mathrm{I}_{2}$.

Four milliliters of sample solution was added to $1 \mathrm{ml}$ reagent A. To this mixture $1 \mathrm{ml}$ of reagent $\mathrm{B}$ was added. Color was allowed to develop for $15 \mathrm{~min}$ at room temperature, and adsorption was read using a spectrophotometer at $535 \mathrm{~nm}$ against a reagent blank (Idris et al. 2007). The membrane solute rejection (SR) is defined as:

$\mathrm{SR}=\left(1-\frac{C_{\mathrm{p}}}{C_{\mathrm{f}}}\right) \times 100$

where, $C_{\mathrm{f}}$ and $C_{\mathrm{p}}$ are the polyethylene glycol concentrations in the feed solution and permeate solution, respectively. The concentration of PEG was determined based on absorbency in a UV-spectrophotometer at a wavelength of $535 \mathrm{~nm}$. Polyethylene glycol (PEG) ca. $500 \mathrm{ppm}$ solutions over a range of specified molecular weights were used for evaluating the selectivity or rejection tests of polysulfone and PSu/PANI nanocomposites membrane and tests were performed at a pressure of 5 bars. Moreover, aqueous solutions of $\mathrm{NaCl} 2,000 \mathrm{mg} / \mathrm{l}$ were used to determine salt rejection rate of membranes. These solutions were adjusted to a pH of $8.5 \pm 0.5$ with $\mathrm{NaOH}$ to simulate conditions similar to seawater.

\section{Result and discussions}

Membrane supporting characterizations

Characterizations are important aspect to understand the performance of materials under service conditions. Progress in characterizing membranes by several advanced equipments such as atomic force microscopy (AFM), scanning electron microscopy (SEM), and transmission electron microscopy (TEM) opens exciting possibilities for the evaluation of membranes to delineate the membrane surface and cross-section morphologies which generally affect membrane performance efficiency. Another important characterization of membrane is mechanical properties that involve membrane behavior under stress and play a major role in membrane performance. Mechanical properties of membranes were studied on Hounsfield H100KS.

\section{Membrane morphologies}

The outer surface and cross-section morphologies of membranes were examined by a scanning electron microscopy. The morphologies for inner and outer surfaces of the polysulfone and PSu/PANI nanocomposite membranes were presented in Figs. $2 \mathrm{a}-\mathrm{c}$ and $3 \mathrm{a}-\mathrm{c}$. Comparing the inner and outer surface structures, it was observed that there were obvious changes in morphology with the addition of polyaniline nanoparticles. The added nanoparticles which resulted in better pores structure as well as stronger interactions on the membranes surface. As shown in the SEM images Fig. 2a-c, nanocomposite membranes had a dense with non-agglomerated surface as well as no phase separation. Dense and non-agglomerated surface might be attributed to good compatibility between polyaniline and polysulfone. However, in the case of cross-section morphologies for polysulfone and nanocomposite, there was a transition from the finger-like structure (Fig. 3a) to very thin skin layer followed by honeycomb structure (Fig. 3b, c. The cross-section morphology change for PSu/PANI nanocomposite might be due to the presence of nanoparticles, which increased the solvent exchange rate during membrane formation. As a consequence, honey comp structure appeared to be very well interconnected, which might be accredited to high permeation rate for nanocomposite membranes. To visualize nanoparticles in dispersions, we used transmission electron microscopy (TEM). Doped polyaniline nanoparticles in dispersions are generally settled or phases separate upon storage due to its poor solubility in common organic solvents, in which case they can be mixed before use. However, undoped polyaniline nanoparticles do not settle down upon storage since they are soluble in organic solvents and they can be dispersed in well manner (like "true" solutions). The morphology of the polyaniline nanoparticles and their dispersions in the polysulfone was ascertained by TEM images. A uniform dispersion of doped polyaniline in conventional polymer using solution processing technique is generally difficult task due to its poor solubility in common organic solvents. However, solubility of HCl-doped PANI can be improved by the using doping/dedoping process where it has been found that undoped polyaniline shows better solubility. 

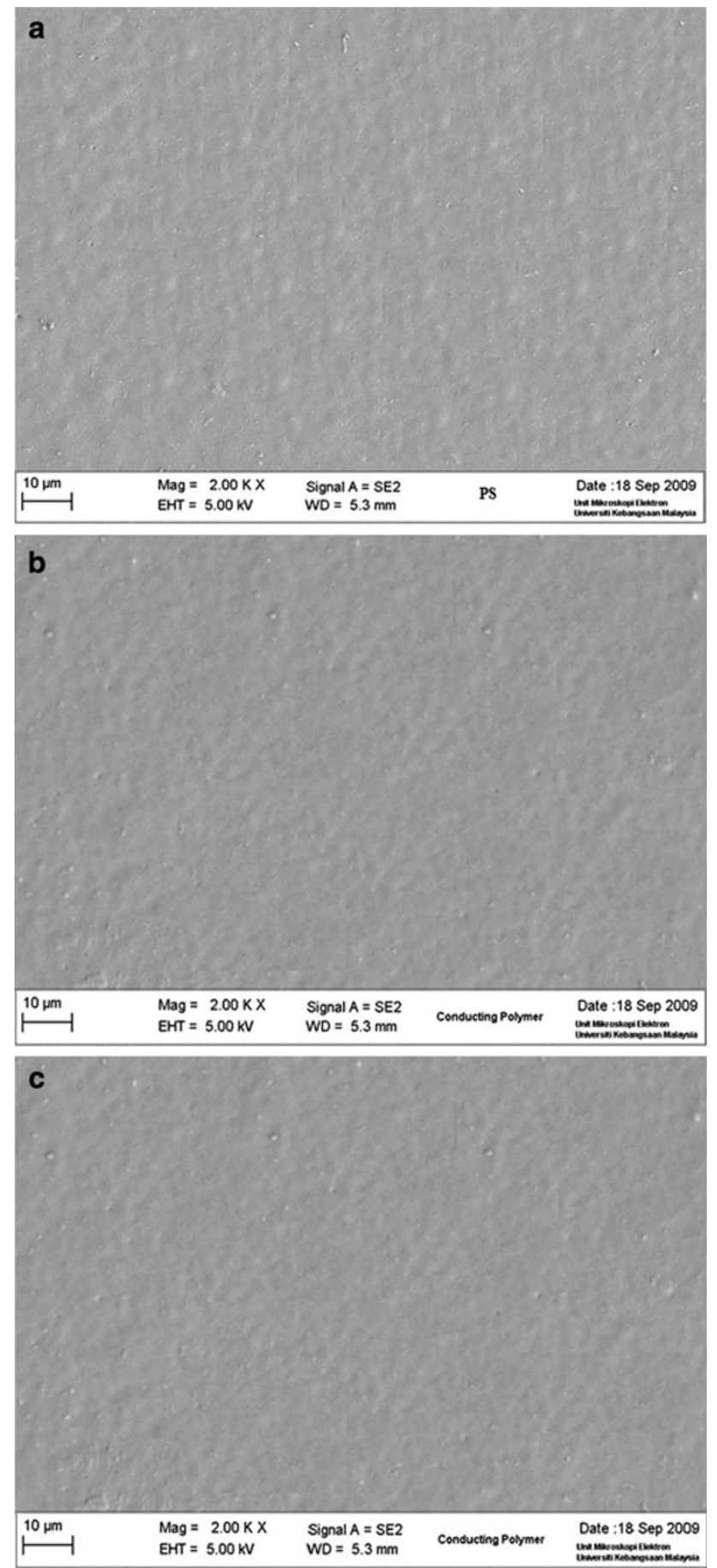

Fig. 2 SEM outer surface morphology of a polysulfone, b undoped PSu/PANI, c doped PSu/PANI nanocomposite membrane

Consequently, TEM micrograph, Fig. 4a, b of PSu/PANI nanocomposite showed the homogeneous mixing which indicated that the polyaniline nanoparticles were well dispersed in the polysulfone matrix. Moreover, PANI nanoparticles displayed spherical structure with the diameter of $\leq 20 \mathrm{~nm}$.

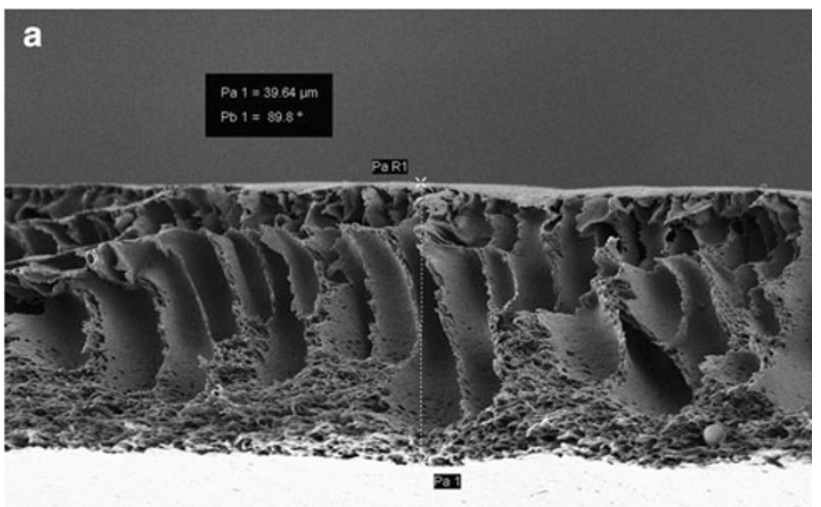

\begin{tabular}{|c|c|c|c|c|}
\hline $10 \mu \mathrm{m}$ & $\begin{array}{l}\mathrm{Mag}=1.00 \mathrm{KX} \\
\mathrm{EHT}=3.00 \mathrm{kV}\end{array}$ & $\begin{array}{l}\text { Signal } A=S E 2 \\
W D=5.2 \mathrm{~mm}\end{array}$ & PS & 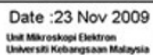 \\
\hline
\end{tabular}
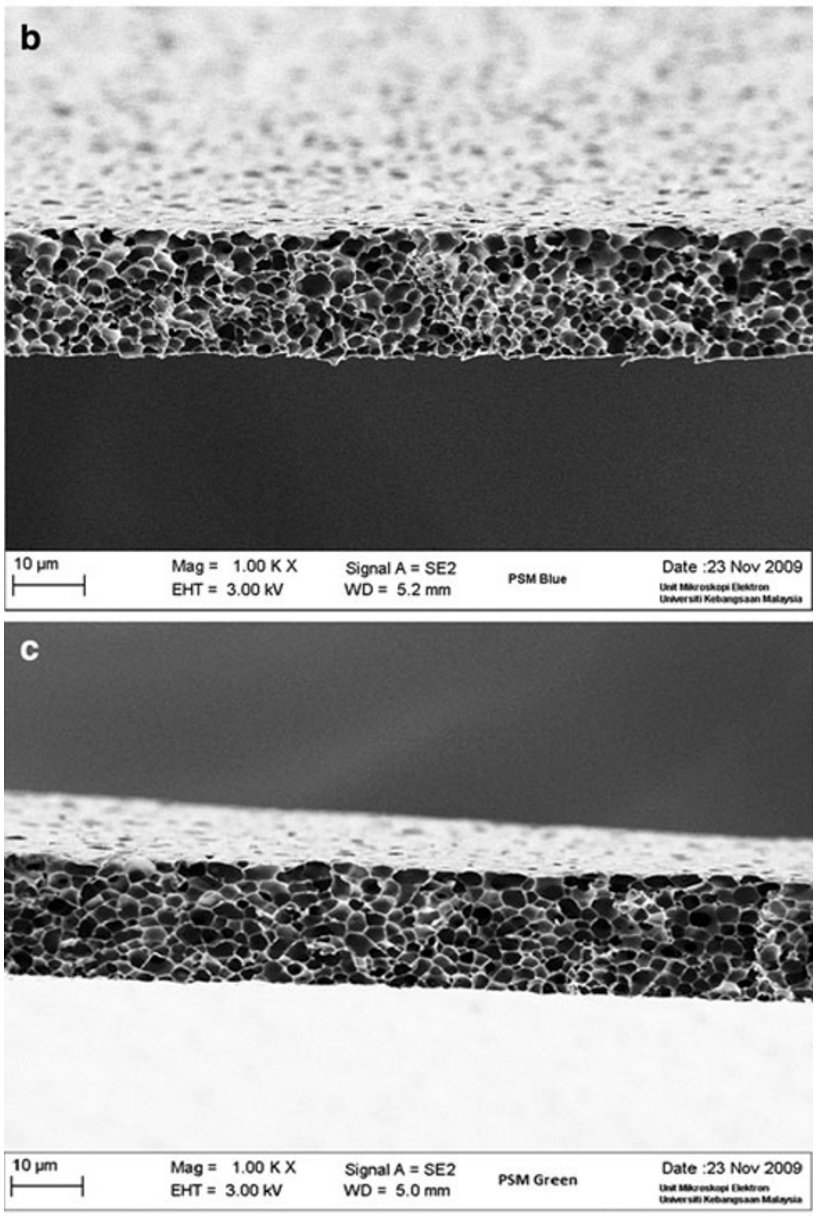

Fig. 3 SEM cross-section morphology of a polysulfone, b undoped PSu/PANI, $\mathbf{c}$ doped PSu/PANI nanocomposite membrane

FTIR-ATR spectra analysis

An ATR-FTIR spectrum of polyaniline in its respective undoped (quinonoid; $\mathrm{NH}-\mathrm{Q}-\mathrm{NH}$ ) and doped state (benzenoid; NH-B-NH) is shown in shows vibration bands at 1,590, 1,498, and 1,305 $\mathrm{cm}^{-1}$, respectively (Furukawa

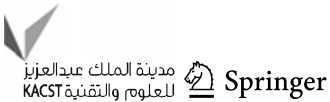




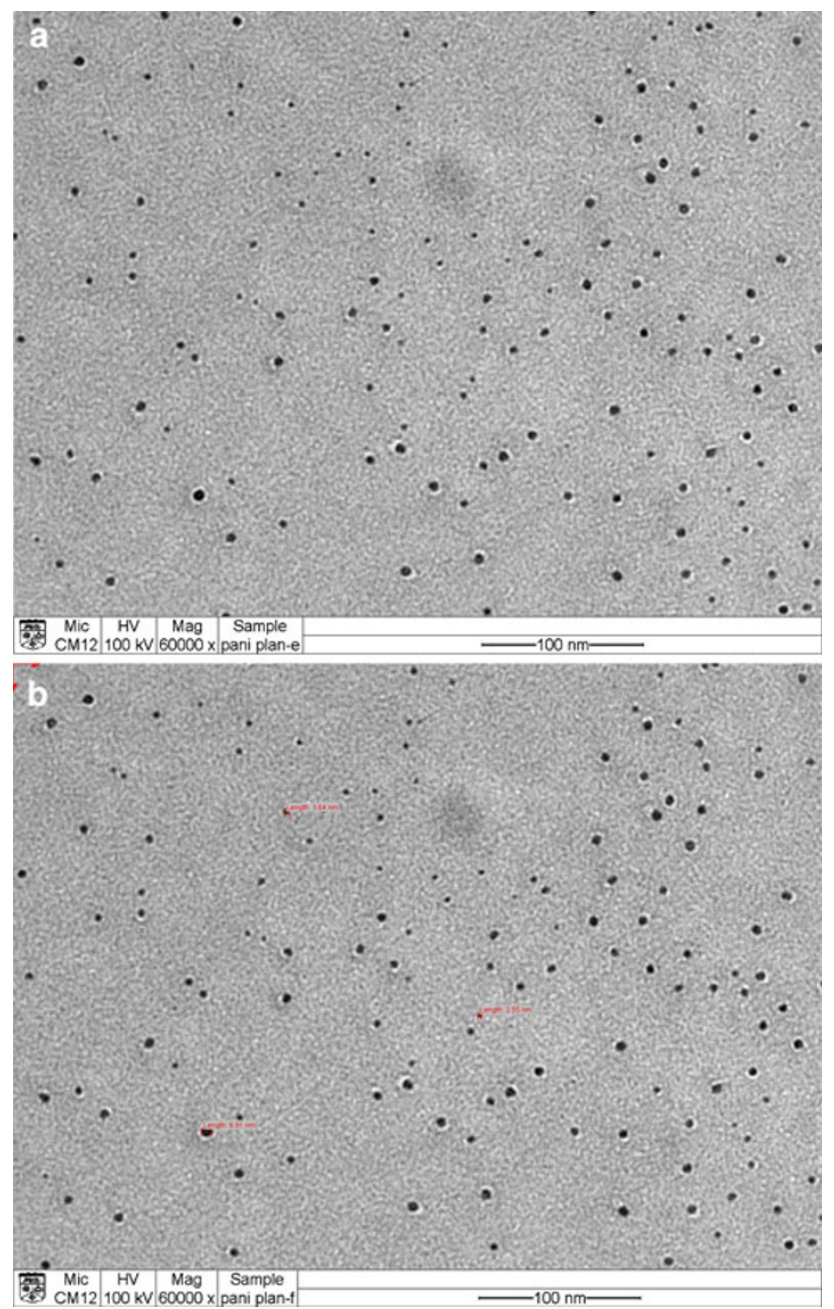

Fig. 4 TEM images of undoped PSu/PANI nanocomposite

et al. 1988; Javed et al. 2007). These values are characteristic peaks of polyaniline-doped and undoped state and are in agreement with theoretical predictions. The band at $1,125.25 \mathrm{~cm}^{-1}$ is due to presence of $\mathrm{C}-\mathrm{N}$ double bond and indicative of protonation. Significantly, the $1,125.25 \mathrm{~cm}^{-1}$ is much pronounced in case of FTIR spectra of doped polyaniline (Fig. 5a). Also, a shift of the band at about 1,580 and $1,508 \mathrm{~cm}^{-1}$ indicate quinoid ring $\mathrm{C}=\mathrm{C}$ stretch and benzenoid ring $\mathrm{C}=\mathrm{C}$ stretch, respectively. Based on this, it can be attributed that all undoped samples have an oxidation degree lower than 0.5 , indicating that the individual chains contain more quinoid rings than benzenoid rings (Fig. 5b). Also, the band at $1,470 \mathrm{~cm}^{-1}$ is dominates the spectrum and is assigned to $\mathrm{C}=\mathrm{N}$ from quinonoid ring. Intensity of the bands at $1,324 \mathrm{~cm}^{-1}$ as well as at $1,262 \mathrm{~cm}^{-1}$ drastically decreases, which may be due to decrease in number of quinonoid rings in the structure (Miroslava and Jaroslav 2011; Takafumi et al. 1987).
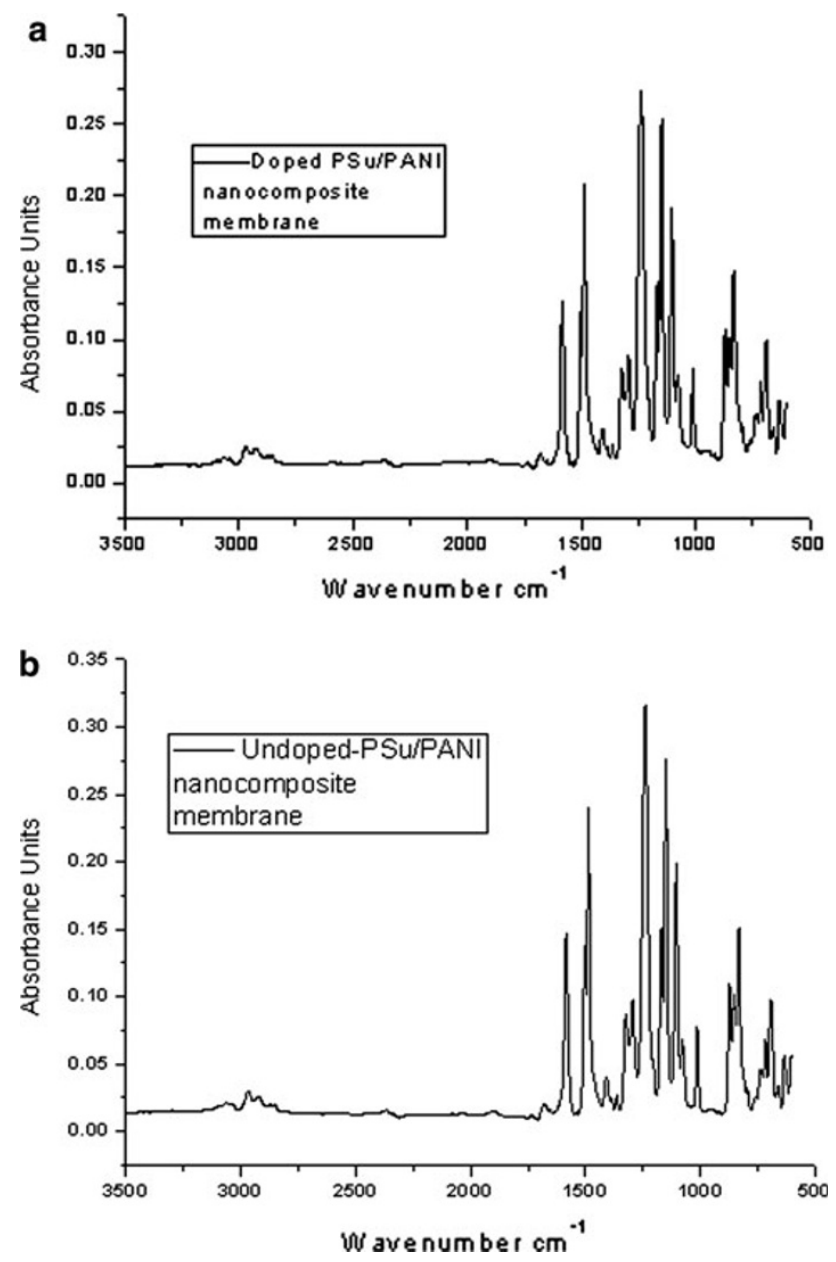

Fig. 5 ATR-FTIR of a PSu, b PSu/PANI nanocomposite membrane

Table 1 Mechanical properties of PSu and PSu/PANI nanocomposite membranes

\begin{tabular}{lcc}
\hline Mechanical properties & $\begin{array}{l}\text { Polysulfone } \\
\text { membrane }\end{array}$ & $\begin{array}{l}\text { PSu/PANI nanocomposite } \\
\text { membrane }\end{array}$ \\
\hline Membrane thickness (mm) & 0.08 & 0.08 \\
Membrane width & 20 & 20 \\
Tensile speed (mm/min) & 20 & 20 \\
Gauge length (mm) & 50 & 50 \\
Tensile (MPa) & 2.688 & 6.44 \\
Elongation at max. (\%) & 4.125 & 16.17 \\
Elongation (\%) & 12.23 & 28.41 \\
\hline
\end{tabular}

\section{Surface roughness}

Atomic force microscopy has been used to characterize the external surface roughness which has been regarded as one of the most important surface properties, and has significant role in membrane permeability and fouling behavior. Membrane surface was achieved by measuring roughness 

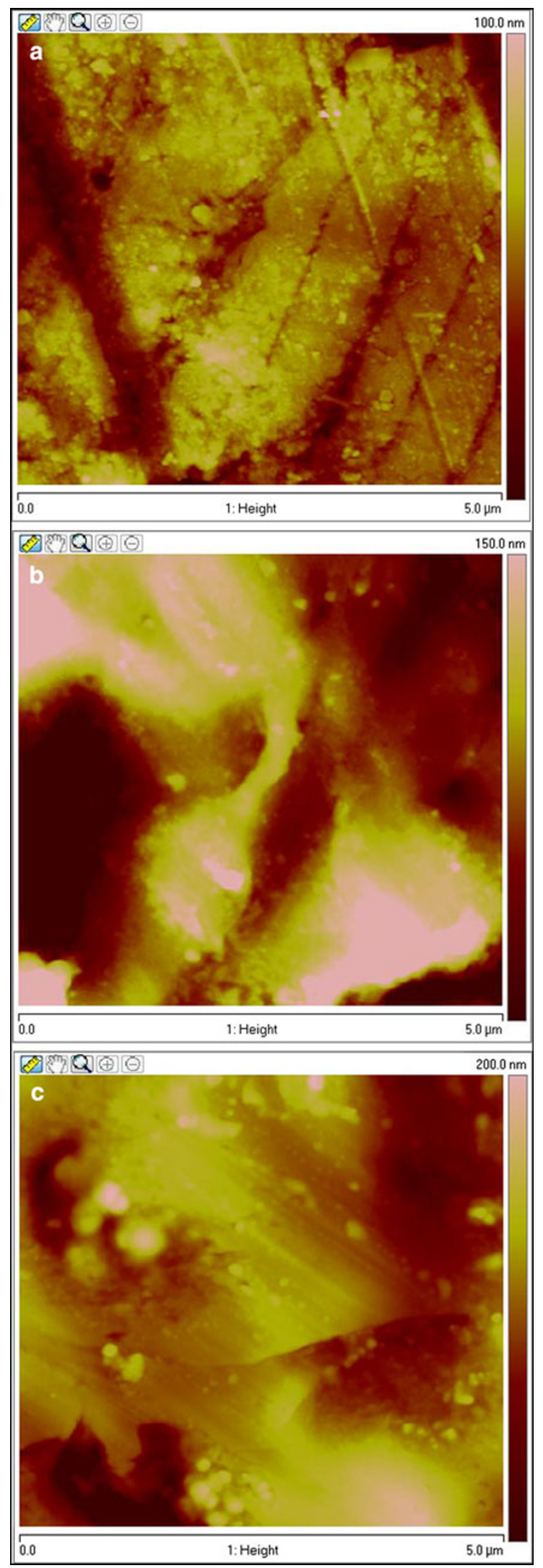

Fig. 6 AFM surface images of a polysulfone, b undoped PSu/PANI, c doped PSu/PANI nanocomposite membranes
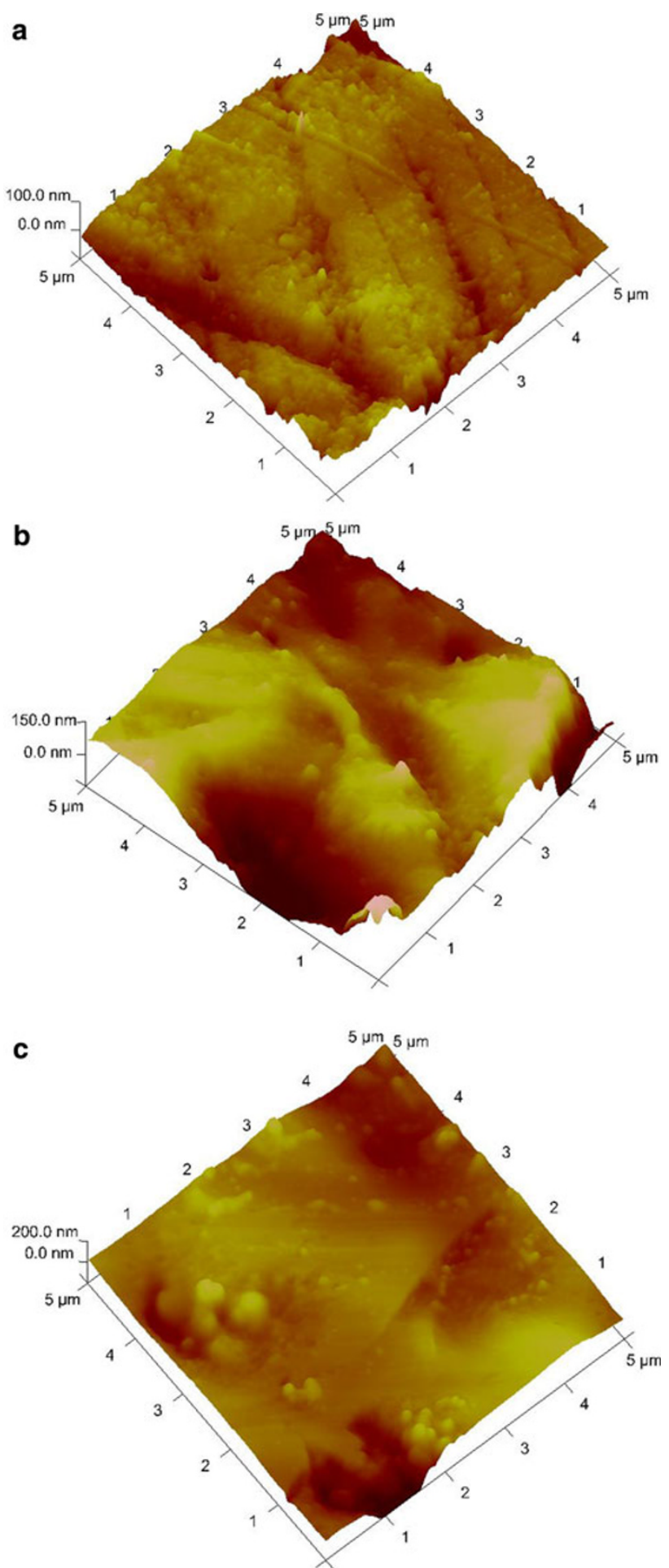

Fig. 7 AFM-3D-images of a polysulfone, b undoped PSu/PANI, c doped PSu/PANI nanocomposite membrane

parameters e.g. the average roughness $\left(R_{\mathrm{a}}\right)$ and root mean square roughness $\left(R_{\mathrm{rms}}\right)$, values of which were given in Table 1. In addition, the AFM phase and 3D images of the membranes, shown in Figs. 6a-c and 7a-c disclosed different extents and occurrences of surface roughness. As a

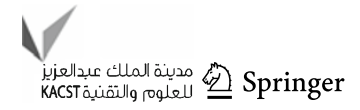


Table 2 Contact angle and roughness of PSu and PSu/PANI nanocomposite membranes

\begin{tabular}{llllll}
\hline Membrane systems & Value of contact angle, $\theta\left(^{\circ}\right)$ & $R_{\mathrm{a}}(\mathrm{nm})(5 \mu \mathrm{m} \times 5 \mu \mathrm{m})$ & $R_{\mathrm{q}}(\mathrm{nm})(5 \mu \mathrm{m} \times 5 \mu \mathrm{m})$ & $\mathrm{SAD}(\%)$ & $\mathrm{ISA}\left(\mu \mathrm{m}^{2}\right)$ \\
\hline Polysulfone & 62 & 45.1 & 57.1 & 2.92 & 25.7 \\
Undoped PSu/PANI & 60 & 28.9 & 40.4 & 2.93 & 25.7 \\
Doped PSu/PANI & 52 & 11.6 & 15.5 & 2.65 & 25.7 \\
\hline
\end{tabular}

result, polysulfone (Fig. 5a) membrane exhibited largescale surface roughness and ridge-and-valley structure which may cause more severe flux decline than in smooth membrane, while the PSu/PANI nanocomposite membrane had uniform smooth surface (Fig. 6b, c). In addition, 3D images of the membranes, Fig. 7a-c clearly showed that nanocomposite membranes had the smoother surface than polysulfone membrane. Therefore, it was observed that inclusion of polyaniline in polysulfone membrane altered the surface roughness properties. Hence it might be ascribed to uniform mixing of polyaniline nanoparticles in polysulfone matrix for the formation of smooth surface of nanocomposite membranes Fig. $7 \mathrm{~b}-\mathrm{c}$.

\section{Mechanical properties}

Mechanical properties that involve membrane behavior under stress play a major role in membrane performance. As a matter of fact, polyaniline alone shows poor mechanical properties. Therefore, it is mostly used either as deposited film or nanofillers (nanoparticles, nanofibers, etc). The tensile strength at break and elongation at break were measured according to the ASTM D412 standard method by a tensile test machine. It was observed that mechanical properties of membrane enhanced after inclusion of polyaniline. Table 1 shows the mechanical properties comparison between polysulfone and composites membranes.

Wettability of membrane surface

For water, a wettable surface can also be explained in terms of hydrophilic surface. The table described varying contact angles and their corresponding solid/liquid interactions. Water contact angles data, which are indicative of wettability of membrane surfaces, were obtained at $25^{\circ} \mathrm{C}$. Pristine PSu and PSu/PANI nanocomposite samples were characterized through contact angle measurement to determine the hydrophilicity for each membrane. The contact angles decreased with the inclusion of polyaniline content which indicated that the addition of a polyaniline can be a useful way to improve membrane hydrophilicity of polysulfone (Table 2). In addition, table showed the variation of the contact angles of doped/undoped polyaniline nanocomposite membranes. As shown in Table 1, it

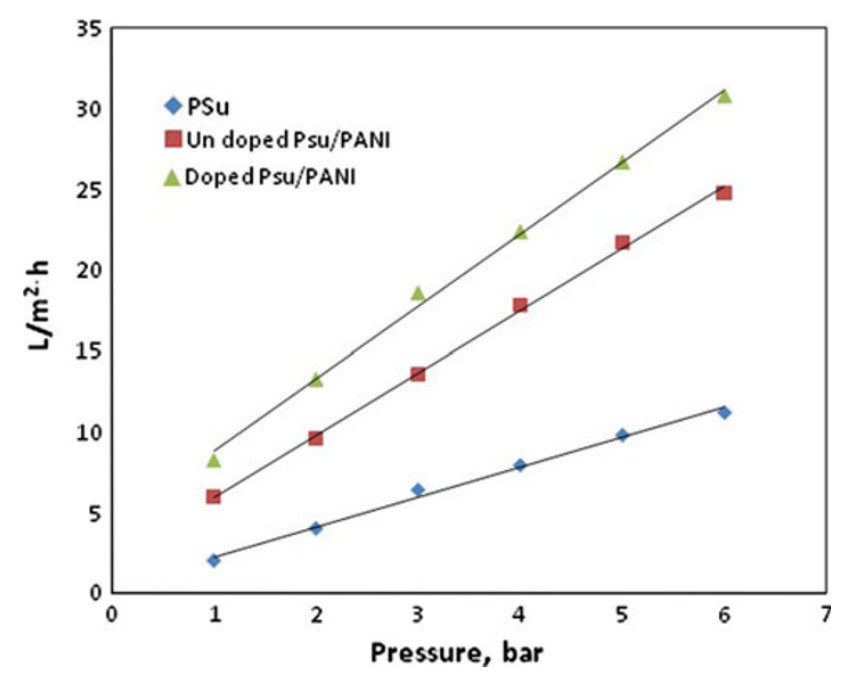

Fig. 8 Pure water flux of PSu, PSu/PANI doped and undoped nanocomposite membranes at $25 \pm 2{ }^{\circ} \mathrm{C}$

Table 3 Salt rejection and MWCO of PSu and PSu/PANI membranes

\begin{tabular}{llc}
\hline Membrane systems & $\begin{array}{l}\text { Rejection rate (\%) of } \\
\mathrm{NaCl}\end{array}$ & MWCO \\
\hline Polysulfone & 93.4 & 580 \\
$\begin{array}{l}\text { Undoped PSu/PANI } \\
\text { nanocomposite }\end{array}$ & 97.3 & 560 \\
$\begin{array}{l}\text { Doped PSu/PANI } \\
\text { nanocomposite }\end{array}$ & 98.6 & 520 \\
\hline
\end{tabular}

was found that the contact angle of doped membranes was higher than the values of undoped PSu/PANI nanocomposite membranes.

\section{Membrane permeability}

In addition, the permeation performance of the membranes was described in experimental section by the water flux, and the solute rejection. Preliminary permeation experiment was conducted for pure water at various ranges of separation pressure (0-6 bars) at temperature $25 \pm 2{ }^{\circ} \mathrm{C}$. Figure 8 shows the variation of flux at different pressures keeping the stirrer speed constant. The pure water flux of the undoped nanocomposite membrane was exhibited 2.8 times higher than that of the PSu substrate membrane 
because PANI was found to be hydrophilic in nature and could be wetted easily by water, while it was found that doped membrane exhibited a higher flux over undoped membrane. This increase in water permeability might be due to the fact that $\mathrm{HCl}$-doped polyaniline enhances the hydrophilicity of the membrane (Liu et al. 1994; Alix et al. 1989). Figure 8 showed that doped PSu/PANI nanocomposite membrane had higher hydrophilicity than undoped membrane and water passed through the doped membrane easily also for all the three membranes acceptable correlation between coefficients $\left(R^{2}=0.98-0.99\right)$ of straight line was obtained. Generally in the pressure-driven physical process, the permeate flux of membranes increases linearly as the trans-membrane pressure increases (Sivakumar et al. 2000). Separation properties of the composite membrane were also tested to verify its application prospect.

\section{Solute rejection}

The salt rejection performance of the membranes is shown in Table 3, where 2,000 mg/l aqueous solution of $\mathrm{NaCl}$ was used as feed. The rejection of sodium chloride for the PSu membrane was 93.4\%, whereas PANI/PSu nanocomposite undoped membrane was found to be $97.3 \%$ rejection of salt. It is revealed that nanocomposite membranes have smaller pore size than pure PSu membrane. However, significant increase in salt rejection was observed for doped $\mathrm{PSu} / \mathrm{PANI}$ nanocomposite membrane. These results indicate that doping modify the network structure of the PSu/ PANI nanocomposite membranes, leading to variation in the membrane transport properties. The addition of nano PANI into the matrix of polysulfone not only increased the hydrophilicity which greatly influenced the rejection of salt. The molecular weight cut-off (MWCO) of the prepared membranes was determined through permeation tests using a group of five PEG, with different molecular weights of $300,600,1,000,1,450$ and 3,350 and 10,000 Da as model solutes. From the rejection behavior, it is found that MWCO of the PSu, PSu/PANI undoped and PSu/PANI doped nanocomposite were about 580, 560 and $520 \mathrm{Da}$, respectively.

\section{Conclusion}

A hydrophilic modification in polysulfone membranes is one of the important concern in the quest for advances and improvements in membrane technology. Concerning this, polyaniline nanoparticles, which enable to change the membrane surface properties during doping/dedoping process were used as nanofillers for the hydrophilic modification of polysulfone membrane. As a result, it was found that polyaniline not only increased the separation characteristics, but also altered the membrane morphology and surface roughness. Therefore, polyaniline nanoparticles inclusion in the polysulfone membrane showed a significant effect on hydrophilic property, which induced the improvement of permeability characteristics of polysulfone membrane.

Open Access This article is distributed under the terms of the Creative Commons Attribution License which permits any use, distribution and reproduction in any medium, provided the original author(s) and source are credited.

\section{References}

Alix A, Lemoine V, Nechtschein M, Travers JP, Mendaro C (1989) Water adsorption study in polyaniline. Synth Metals 29:E457E462

Anadão P, Sato LF, Wiebeck H, Valenzuela-Díaz FR (2010) Montmorillonite as a component of polysulfone nanocomposite membranes. Appl Clay Sci 48:127-132

Ashraf MW (2009) Utilization of supported liquid membrane technique: removal of chemical species from industrial waste effluents. VDM. ISBN:978-3-639-12996-0

Baker R W (2004) Membrane technology and applications. Wiley, pp 538

Bhadra S, Khastgir D, Singha NK, Lee JH (2009) Progress in preparation, processing and applications of polyaniline. Prog Polym Sci 34:783-810

Deligöz H (2007) Preparation of self-standing polyaniline-based membranes: doping effect on the selective ion separation and reverse osmosis properties. J Appl Polym Sci 105:2640-2645

Deng H, Xu Y, Chen Q, Wei X, Zhu B (2011) High flux positively charged nanofiltration membranes prepared by UV-initiated graft polymerization of methacrylatoethyl trimethyl ammonium chloride (DMC) onto polysulfone membranes. J Membr Sci 366:363-372

Diallo M, Duncan J, Savage N, Street A, R Sustich (2009) Nanotechnology applications for clean water, 1st edn. Elsevier

Dong HB, Xu Y, Yi Z, Shi J (2009) Li Modification of polysulfone membranes via surface-initiated atom transfer radical polymerization. Appl Surf Sci 255:8860-8866

Fan Z, Wang Z, Sun N, Wang J, Wang S (2008) Performance improvement of polysulfone ultrafiltration membrane by blending with polyaniline nanofibers. J Membr Sci 363-371

Fan Z, Wang Z, Duan M, Wang J, Wang S (2008b) Preparation and characterization of polyaniline/polysulfone nanocomposite ultrafiltration membrane. J Membr Sci 310:402-408

Feldheim DL, Elliott CM (1992) Switchable gate membranes. Conducting polymer films for the selective transport of neutral solution species. J Membr Sci 70:9-15

Furukawa Y, Ueda F, Hyodo Y, Harada I, Nakajima T, Kawagoe T (1988) Vibrational spectra and structure of polyaniline. Macromolecules 21:1297-1305

Ghosh AK, Hoek EM V (2009) Impacts of support membrane structure and chemistry on polyamide-polysulfone interfacial composite membranes. J Membr Sci 336:140-148

Gupta Y, Wakeman R, Hellgardt K (2006) High-productive, nanostructured polyaniline membranes for gas separation. Desalination 199:474-476

He D, Susanto H, Ulbricht M (2009) Photo-irradiation for preparation, modification and stimulation of polymeric membranes. Prog Polym Sci 34:62-98 
Hegde C, Isloor AM, Padaki M, Wanichapichart P, Liangdeng Y (2011) Synthesis and desalination performance of $\mathrm{Ar}^{+}-\mathrm{N}^{+}$ irradiated polysulfone based new NF membrane. Desalination 265:153-158

Higuchi A, Mishima S, Nakagawa T (1991) Separation of proteins by surface modified polysulfone membranes. J Membr Sci 57:175-185

Hoek EMV, Ghosh AK (2009) Nanotechnology-based membranes for water purification. In: Savage $\mathrm{N}$ et al (eds) Nanotechnology applications for clean water. William Andrew Inc., Norwich, pp 47-58

Huang JX, Kaner RB (2004) Nanofiber formation in the chemical polymerization of aniline: a mechanistic study. Angew Chem Int Ed 43:5817-5821

Idris A, Zain NM, Noordin MY (2007) Synthesis, characterization and performance of asymmetric polyethersulfone (PES) ultrafiltration membranes with polyethylene glycol of different molecular weights as additives. Desalination 207:324-339

Jadav GL, Singh PS (2009) Synthesis of novel silica-polyamide nanocomposite membrane with enhanced properties. J Membr Sci 328:257-267

Javed A, Ufana R, Sharif A (2007) Effect of ferrofluid concentration on electrical and magnetic properties of the Fe3O4/PANI nanocomposites. J Magn Magn Mater 314:93-99

Jeong BH, Hoek MVE, Yan Y, Subramani A, Huang X, Hurwitz G, Ghosh AK, Jawor A (2007) Interfacial polymerization of thin film nanocomposites: a new concept for reverse osmosis membranes. J Membr Sci 294:1-7

Lee KP, Arnot TC, Mattia D (2011) A review of reverse osmosis membrane materials for desalination-development to date and future potential. J Membr Sci 370:1-22

Liu MJ, Tzou K, Gregory RV (1994) Influence of the doping conditions on the surface energies of conducting polymers. Synth Metal 63:67-71
Mansouri J, Burford RP (1994) Novel membranes from conducting polymers. J Membr Sci 87:23-34

Miroslava T, Jaroslav S (2011) Polyaniline: the infrared spectroscopy of conducting polymer nanotubes (IUPAC Technical Report). Pure Appl Chem 83:1803-1817

Mulder M, Mulder J (1996) Basic principles of membrane technology, 2nd edn. Springer

Nunes SP, Peinemann KV (2010) Advanced polymeric and organicinorganic membranes for pressure-driven processes. Compr Membr Sci Eng 1.06:113-129

Patel R, Im SJ, Ko YT, Kim JH, Min BR (2009) Preparation and characterization of proton conducting polysulfone grafted poly (styrene sulfonic acid) polyelectrolyte membranes. J Ind Eng Chem 15:299-303

Rebattet L, Escoubes M, Genies E, Pinern M (1995) Effect of doping treatment on gas transport properties and on separation factors of polyaniline membranes. J Appl Polym Sci 57:1595-1604

Sabde AD, Trivedi MK, Ramachandhran V, Hanra MS (1997) Casting and characterization of cellulose acetate butyrate based UF membranes. Desalination 114:223-232

Sivakumar M, Malaisamy R, Sajitha CJ, Mohan D, Mohan V, Rangarajan R (2000) Preparation and performance of cellulose acetate-polyurethane blend membranes and their applicationsII. J Memb Sci 169:215-228

Sivakumar M, Mohan D, Rangarajan R (2006) Studies on cellulose acetate-polysulfone ultrafiltration membranes: II. Effect of additive concentration. J Membr Sci 268:208-219

Takafumi H, Yoshinori H, Hajime T, Toshio N (1987) Structure and electronic properties of polyaniline. Jpn J Appl Phys 26:L1803L1805

Ulbricht M (2006) Advanced functional polymer membranes. Polymer 22:2217-2262

Wen L, Kocherginsky NM (1999) Doping-dependent ion selectivity of polyaniline membranes. Synth Metals 106:19-27 